\title{
Output-Only Modal Analysis Based on Improved Empirical Mode Decomposition Method
}

\author{
Shiqiang Qin, Qiuping Wang, and Juntao Kang \\ School of Civil Engineering and Architecture, Wuhan University of Technology, Wuhan 430070, China \\ Correspondence should be addressed to Juntao Kang; jtkang@163.com
}

Received 27 May 2015; Accepted 15 September 2015

Academic Editor: Giovanni Berselli

Copyright (C) 2015 Shiqiang Qin et al. This is an open access article distributed under the Creative Commons Attribution License, which permits unrestricted use, distribution, and reproduction in any medium, provided the original work is properly cited.

\begin{abstract}
The output-only modal analysis for bridge structures based on improved empirical mode decomposition (EMD) is investigated in this study. First, a bandwidth restricted EMD is proposed for decomposing nonstationary output measurements with close frequency components. The advantage of bandwidth restricted EMD to standard EMD is illustrated by a numerical simulation. Next, the modal parameters are extracted from intrinsic mode function obtained from the improved EMD by both random decrement technique and stochastic subspace identification. Finally, output-only modal analysis of a railway bridge is presented. The study demonstrates the mode mixing issues of standard EMD can be restrained by introducing bandwidth restricted signal. Further, with the improved EMD method, band-pass filter is no longer needed for separating the closely spaced frequency components. The modal parameters extracted based on the improved EMD method show good agreement with those extracted by conventional modal identification algorithms.
\end{abstract}

\section{Introduction}

Monitoring, maintenance, and management at operation stage play an important role in the whole life span of largescale bridge structures. Damage detection in time and proper repairing measures prolong the service life of bridge. Thus, the structural health monitoring (SHM) of bridge structures has gained more and more concern by both researchers and practicing engineers. By installing various sensors on bridge, mechanical behaviors and dynamic responses of bridge are recorded under normal load conditions (ambient, vehicles, etc.) and extreme load conditions (hurricane, earthquake, etc.). In this way, structural health condition can be assessed resulting in good advices for maintenance and management. Besides, the recorded data can be further used to validate and improve the design theory of bridge with nonconventional structure system. Successful applications of SHM for bridge structures can be found in [1-6] and the references therein.

A relevant research topic in SHM is to experimentally determine the dynamic characteristics with high accuracy. For bridge structures, the basic dynamic properties are the modal parameters, that is, natural frequencies, damping ratios, and mode shapes. These modal properties are the inherent properties of structure, only related with mass distribution, stiffness, and boundary condition. Variation of modal parameter implies change in the inherent properties of structure, by which structural damage can be detected. Therefore, modal parameters are often served as indicators in modal-based damage detection techniques $[7,8]$. In addition, experimentally determined modal parameters are regarded as key parameters for updating and calibrating the preliminary finite element (FE) model developed from design documents [9-12]. Served as baseline for SHM, the updated FE model can be used to predict the dynamic responses and damage detection of bridge. Furthermore, the modal parameters are important for condition assessment; see [13], for example. Traditionally, modal analysis is often performed based on both input and output measurements of structures, meaning special excitation devices, such as drop weights, shakers, are needed. However, these requirements are difficult to be fulfilled for large-scale bridge structures. This is because for one thing, bridge structure cannot be fully excited by only one 
or several excitation devices; moreover, these devices are very expensive for large-scale application. For the other, the operation service needs to be interrupted. Therefore, it is more meaningful to extract modal parameters from operational vibration measurements, namely, output-only modal analysis or operational modal analysis (OMA). At present, there are several sophisticated approaches for output-only modal analysis of bridge structures. These OMA approaches can be grouped into two categories, namely, frequency domain methods and time domain methods. The typical frequency domain methods include Pick-Peaking (PP) from the power spectra density [14], frequency domain decomposition (FFD) [15], and the operational PolyMax [16]. The common used time domain methods contain auto regressive moving averaging (ARMA) method [17], the natural excitation method (NExT) [18], the least square complex exponential (LSCE) method [19], the Ibrahim time domain (ITD) method [20], and the stochastic subspace identification (SSI) [21].

Two commonly used assumptions of output-only modal analysis algorithms are the white noise assumption of unmeasured input excitations as well as the stationary process assumption of the outputs. For example, the ambient excitations are assumed to be white noise in SSI algorithm so that they can be combined with test inaccuracies and modelling errors. Thus, the stochastic state space model is obtained from the discrete state space model. However, operational vibration measurements are not always stationary signals. Although the existing output-only modal analysis algorithms yield satisfactory results, it is still worthy investigating alternative approaches for modal identification with nonstationary output-only responses, especially from theoretical perspective.

Several examples of output-only modal analysis performed in large civil structures have been reported in technical literature, of which the most relevant cases regarding nonstationary operational vibration measurements are presented herein. Yang et al. [22, 23] proposed a modal identification method based on Hilbert-Huang transform (HHT) in conjunction with band-pass filter using measured free vibration time histories. Yang's study also pointed out that limited by poor frequency resolution of wavelet transform (WT), the identified results from HHT seem to be more accurate than those from WT. He et al. [24] extracted the modal parameters of Nanjing Railway Bridge based on empirical mode decomposition (EMD) and random decrement technique (RDT). Yu and Ren [25] proposed an EMD based SSI modal identification method, which reduced the postprocessing work in SSI algorithm (picking the stable poles). Band-pass filter was also employed to treat the closely spaced modes. Yan and Miyamoto [26] made a comparative study between HHT and WT for modal analysis. The results found that for well-separated modes both methods are applicable, whereas for close spaced modes, the WT method is more effective. Abovementioned literatures proved that it is possible to extract modal parameters from nonstationary operational vibration measurements, but more attention should be paid to closely spaced modes. The bridge structures, often large in scale, fall into the category has low frequencies and close modes. Although some useful algorithms such as ensemble
EMD (EEMD) [27] and the masking signal method [28] have been proposed to deal with close spaced modes, it is still worthy to investigate this problem, especially that EMD is often combined with time domain algorithms to identify modal parameters.

This paper focuses on output-only modal analysis of bridge structure based on an improved EMD method. The bandwidth restricted EMD is proposed to deal with closely spaced modes. Without band-pass filter, the closely spaced modes can be well separated with bandwidth restricted EMD. Both RDT and SSI algorithm are employed to identify modal parameters when the single frequency component of original signal is obtained. The text of this paper is organized as follows: in Section 2, the improved EMD method is introduced. A numerical simulation is used to illustrate the effectiveness of bandwidth restricted EMD in decomposing signals with close frequency components. Section 3 explains the basic principles of extracting modal parameters from intrinsic mode functions by RDT and SSI. The output-only modal analysis of a railway bridge based on improved EMD is accomplished in Section 4. The PP and SSI algorithms are used for comparison. Conclusions are made at last.

\section{An Improved EMD Method}

2.1. Standard EMD. The EMD, first proposed by Huang et al. [29], is an effective approach to decompose nonstationary, multicomponent signal. It can decompose multicomponent signal into a series of intrinsic mode functions (IMFs) and a residual. By definition an IMF has to satisfy the following two conditions:

(1) In the whole data set, the number of extremes and the number of zero crossings may differ but no more than one; (2) At any point, the local average is zero.

For an arbitrary time signal, the basic steps of EMD are as follows.

(a) Sifting process: Find all the extremes of $x(t)$. Define the upper and lower envelope of $x(t)$, which can be obtained through connecting all the local maxima and local minima with cubic spline curve, respectively. Calculate the mean value of the two envelopes. The difference between the $x(t)$ and the mean value is designated as $h_{1}(t)$.

(b) Checking process: Check whether $h_{1}(t)$ satisfies the two conditions of IMF. If $h_{1}(t)$ is an IMF, then take $c_{1}(t)=h_{1}(t)$ as the first IMF of $x(t)$. Otherwise, let $x(t)=h_{1}(t)$ and repeat the sifting process until the first IMF is found out.

(c) Looping process: Define $r_{1}(t)=x(t)-c_{1}(t)$ as the new signal and repeat (a), (b) until $r_{n}(t)$ is smaller than a predetermined threshold. Then, the original signal $x(t)$ can be rewritten as

$$
x(t)=\sum_{i=1}^{n} c_{i}(t)+r_{n}(t),
$$




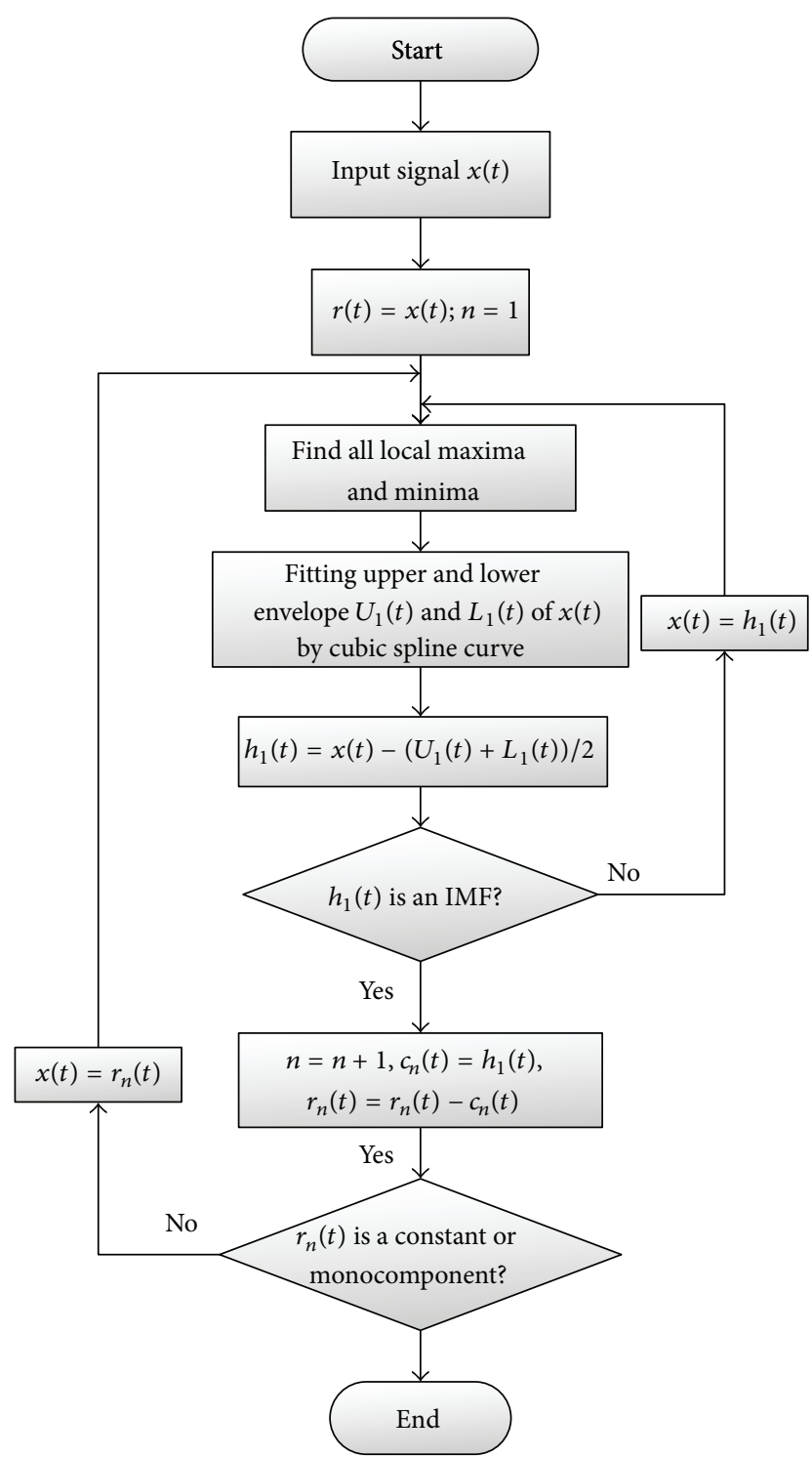

Figure 1: The flow chart of standard EMD.

where $c_{i}(t)(i=1,2,3, \ldots, n)$ represents the IMFs of the $x(t)$ from high frequency to low frequency component. $r_{n}(t)$ is a single value function and represents the residue of $x(t)$. The flow chart of standard EMD is shown in Figure 1. Ideally, each IMF should only contain one frequency component, but in practice, it is often the case that one IMF contains several frequency components. This phenomenon is called mode mixing. It will be further discussed in the next section.

2.2. Bandwidth Restricted EMD. As discussed in Section 2.1, EMD is an effective signal processing method especially when dealing with nonlinear and nonstationary signals. But it still faces some big challenges. Mode mixing is one of these challenges. It means that one IMF contains multifrequency components or one frequency component is decomposed into several IMFs. Under the following two conditions, it may lead to mode mixing during EMD:

(1) The intermittency of signal: This means that the signal has intermittency frequency components. In this case, several different frequency components will be decomposed into one IMF. Figure 2 gives an example of mode mixing because of intermittency of signal. $x_{1}(t)$ and $x_{2}(t)$ are two frequency components of signal $x(t)$, and imf1 and imf 2 are the IMFs obtained by applying standard EMD to $x(t)$. It is obvious that because of intermittency of $x_{1}(t)$, both imf1 and imf 2 contain multifrequency components.

(2) The closely spaced frequency components of signal: In this case, one frequency component will be decomposed into several IMFs. Figure 3 shows an example of this kind of mode mixing. $x_{1}(t)$ and $x_{2}(t)$ are two sinusoidal waves with the frequency of $1.8 \mathrm{~Hz}$ and $2.5 \mathrm{~Hz}$, respectively. imf1 and imf 2 are two IMFs obtained from standard EMD. It is obvious that the two frequency components are decomposed into both IMFs.

In this paper, a bandwidth restricted EMD is proposed to deal with the problem of mode mixing. It has the advantage of easy implementation and does not need the a priori knowledge of the signal to be analyzed. From the theory background of EMD, it is known that each IMF is obtained by subtracting the mean value of upper bound and lower bound of original signal, meaning that EMD has a property similar to binary filter. The proposed method is based on this property and can be realized as the following steps:

(1) Suppose $x(t)$ is a time history signal. Apply standard EMD to $x(t)$, set stop criterion options to make it decompose $x(t)$ into only one IMF and a residual; that is,

$$
x(t)=\mathrm{IMF}_{1}(t)+r_{1}(t) .
$$

(2) Calculate the instant amplitude $a_{1}(i)$ and instant frequency $f_{1}(i)$ of $\operatorname{IMF}_{1}(t)$ based on Hilbert transform. The frequency content of bandwidth restricted signal is set as

$$
f=A_{0} \times \frac{\sum_{i=1}^{k} a_{1}(i) f_{1}^{2}(i)}{\sum_{i=1}^{k} a_{1}(i) f_{1}(i)},
$$

where $A_{0}$ is bandwidth restricted signal coefficient.

(3) Construct the bandwidth restricted signal $s(t)$ by

$$
s(t)=B \sin (2 \pi f t),
$$

where $B$ is a constant; it can be selected according to the amplitude of $x(t)$.

(4) Apply standard EMD to $y(t)=x(t)+s(t)$, making that it only decomposes one IMF and a residual

$$
y(t)=\mathrm{IMF}_{1,1}(t)+r_{1,1}(t) .
$$



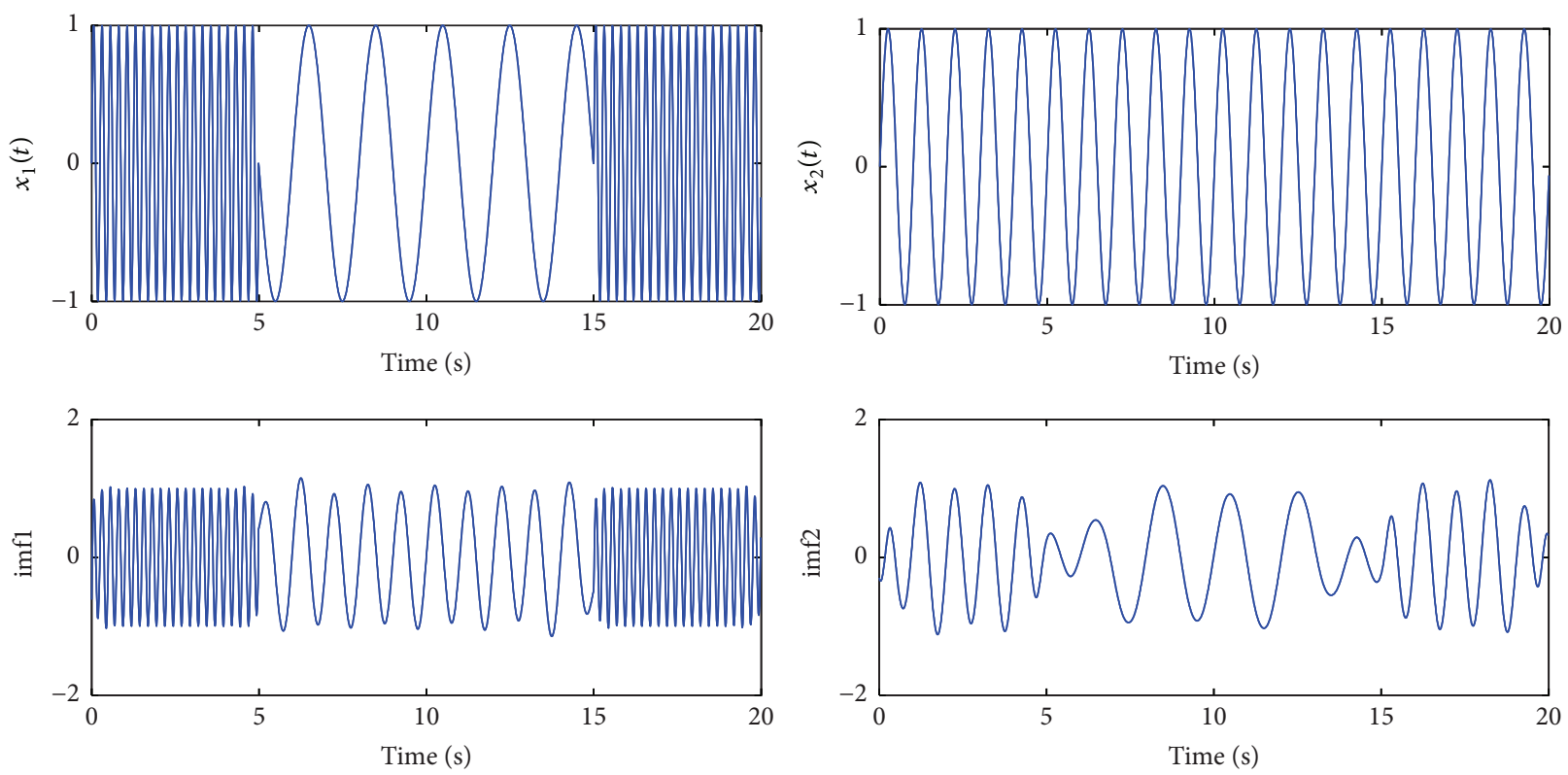

FIGURE 2: Mode mixing due to intermittency of signal. $x_{1}(t)$ and $x_{2}(t)$ are the two frequency components of $x(t)$. imf1 and imf2 are the two IMFs obtained from standard EMD of $x(t)$.
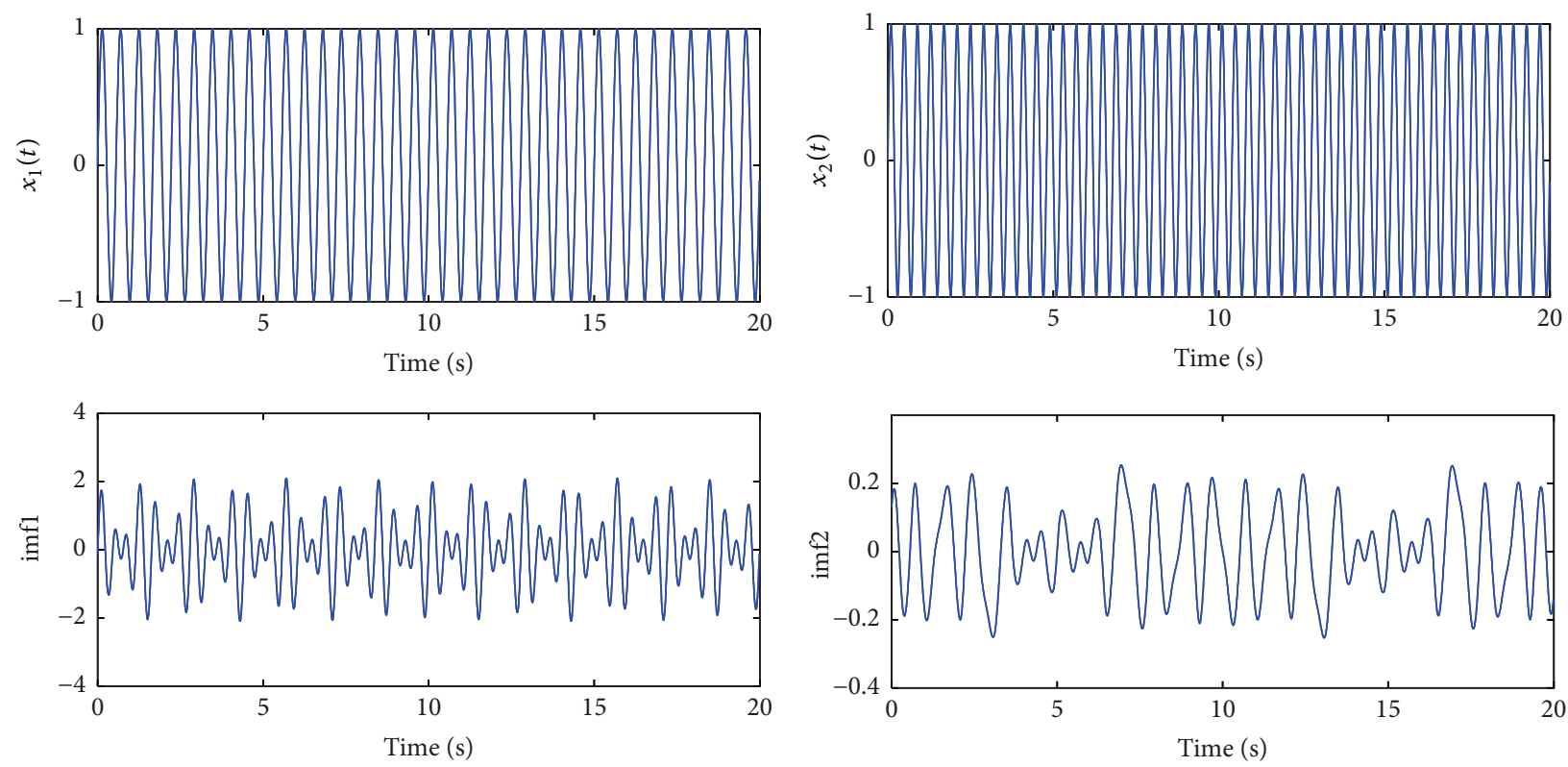

FIGURE 3: Mode mixing due to closely spaced frequency components of signal. $x_{1}(t)$ and $x_{2}(t)$ are the two frequency components of $x(t)$. imf1 and imf2 are the two IMFs obtained from standard EMD of $x(t)$.

(5) Check whether $h_{1}(t)=\operatorname{IMF}_{1,1}(t)-s(t)$ leads to mode mixing. If not, take $h_{1}(t)$ as the first IMF of $x(t)$. Otherwise, take $h_{1}(t)$ as $x(t)$ and repeat (1) (4) for $n$ times until $h_{1, n}(t)$ do not lead to mode mixing any more. In order to check whether an IMF leads to mode mixing, the frequency distribution of the IMF obtained from Hilbert transform can be employed.

(6) Repeat (1) (5) until all IMFs of $x(t)$ are extracted.
Figure 4 shows the flow chart of bandwidth restricted EMD.

Numerical Simulation. To evaluate the effect of bandwidth restricted EMD, a simulation example is presented here. The simulation signal $y(t)$ is composed of three sinusoidal waves with frequency of $10 \mathrm{~Hz}, 15 \mathrm{~Hz}$, and $18 \mathrm{~Hz}$. It can be expressed as $y(t)=\sin (20 \pi t)+\sin (30 \pi t)+\sin (36 \pi t)$. The sampling frequency is $200 \mathrm{~Hz}$ and the sampling time is $2.5 \mathrm{~s}$. Figure 5 


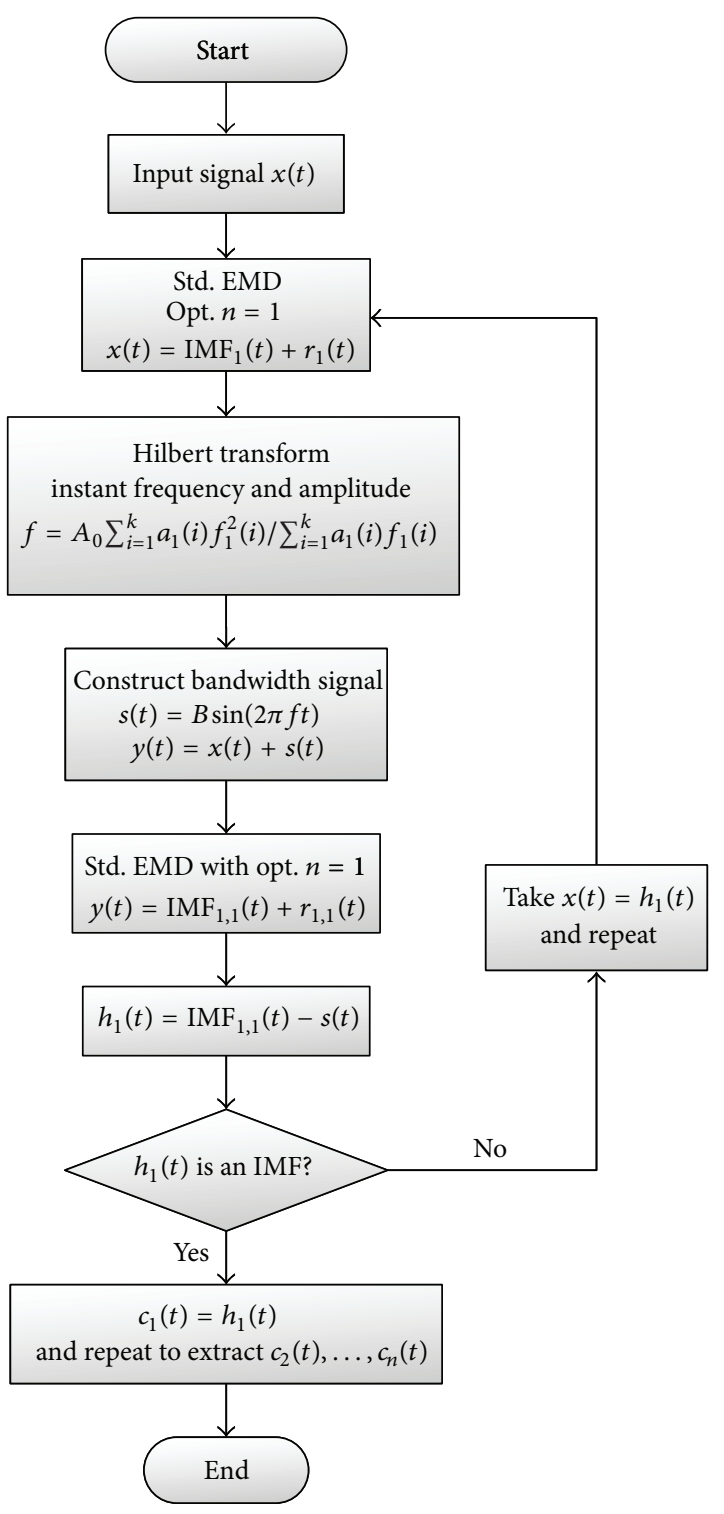

FIGURE 4: The flow chart of bandwidth restricted EMD.

shows the three IMFs obtained from EMD $\left(C_{1} \sim C_{3}\right)$ and from bandwidth restricted EMD (BR-C $\sim$ BR- $C_{3}$ ). It is clearly shown in the figure that $C_{1} \sim C_{3}$ lead to mode mixing while BR- $C_{1} \sim$ BR- $C_{3}$ contain only one frequency component. The simulation example shows that the bandwidth restricted EMD effectively solved the problem of mode mixing.

\section{Modal Parameter Identification Using IMFs}

By introducing bandwidth restricted EMD, the mode mixing of standard EMD is effectively restrained. The obtained IMF is single frequency component, which can be seen as the dynamic response of a single degree of freedom (SDOF) system. Thus, the remaining problem is to identify modal parameters from SDOF system. Both RDT and SSI can be used to extract modal parameters from IMFs.

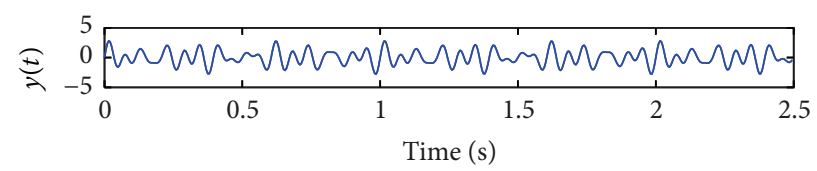

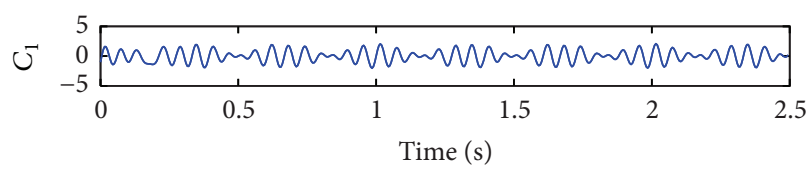$$
\text { Time (s) }
$$
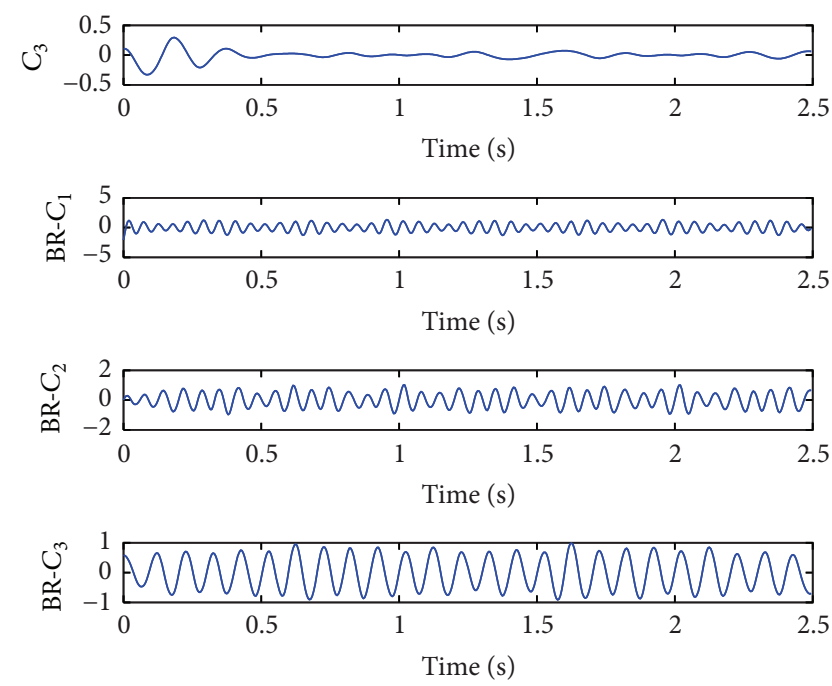

FIGURE 5: The comparison of IMFs obtained from EMD $\left(C_{1} \sim\right.$ $C_{3}$ ) and bandwidth restricted EMD (BR-C $\left.C_{1} \sim \mathrm{BR}-C_{3}\right), y(t)$ is the simulated original signal.

3.1. Random Decrement Technique. RDT is a time domain signal processing approach that is developed for extracting free decay response from stationary linear structure vibration response. Like NExT and LSCE method mentioned in Section 1, RDT is also a time domain technique. These modal identification techniques, including RDT, NExT, and LSCE, are also capable of dealing with closely spaced modes $[18,19]$. However, EMD based RDT modal identification technique is more suitable for large-scale structures since both excitations and responses are nonstationary processes.

The basic principles of RDT are described as follows. For a SDOF linear vibration system, the displacement response $x(t)$ is composed of three parts. The first part results from initial displacement, the second is from initial velocity, and the third is from random excitations. Thus, $x(t)$ can be expressed as

$$
x(t)=x(0) D(t)+\dot{x}(0) V(t)+\int_{0}^{t} h(t-\tau) f(\tau) d \tau,
$$

where $x(0)$ and $\dot{x}(0)$ are the initial displacement and initial velocity, respectively. $D(t)$ is the free vibration response of the system with initial displacement being 1 and initial velocity being $0 . V(t)$ is the free vibration response with initial displacement being 0 and initial velocity being $1 . f(\tau)$ is 
the random excitations at time segment $\tau . h(t)$ is unit impulse response function of SDOF system. If the stochastic part can be separated and eliminated from $x(t)$, one can expect the free vibration part of $x(t)$. Select a reasonable value $A$ to intercept the displacement response $x(t)$, the crossing points are denoted as $\left(t_{i}, x\left(t_{i}\right)\right)(i=1,2,3, \ldots)$. A series of subsample functions $x\left(t-t_{i}\right)$ can be obtained through moving the start time to the crossing points,

$$
\begin{aligned}
x\left(t-t_{i}\right)= & x\left(t_{i}\right) D\left(t-t_{i}\right)+\dot{x}\left(t_{i}\right) V\left(t-t_{i}\right) \\
& +\int_{t_{i}}^{t} h(t-\tau) f(\tau) d \tau .
\end{aligned}
$$

In output-only modal analysis, the random excitations are assumed to be stationary Gaussian white noise. Therefore, the change of start time will not change the stochastic characteristic of random excitation functions. Thus, a series of random process can be obtained from

$$
x_{i}(t)=A D(t)+\dot{x}\left(t_{i}\right) V(t)+\int_{0}^{t} h(t-\tau) f(\tau) d \tau .
$$

Since $\dot{x}\left(t_{i}\right)$ is a zero-mean stationary Gaussian process, hence, $E\left[\dot{x}\left(t_{i}\right)\right]=0$ and $E[f(t)]=0$. The mathematical expectation $E\left[x_{i}(t)\right]$ is obtained by

$$
\begin{aligned}
E\left[x_{i}(t)\right]= & A D(t)+E\left[\dot{x}\left(t_{i}\right)\right] V(t) \\
& +\int_{0}^{t} h(t-\tau) E[f(\tau)]=A D(t),
\end{aligned}
$$

which represents the free vibration response with initial displacement being $A$ and initial velocity being 0 .

After obtaining the free decays of IMF, the modal parameter can be estimated by Hilbert transform. Without loss of generality, the free vibration response in (9) can be rewritten as

$$
x(t)=A_{0} e^{-\xi \omega_{0} t} \cos \left(\omega_{d} t+\phi_{0}\right)
$$

where $\omega_{0}$ is the circular frequency and $\omega_{d}$ is the damping frequency. $\xi$ is the damping ratio and $A_{0}$ is a constant. The corresponding analytical expression of $x(t)$ is obtained by

$$
z(t)=x(t)+i \tilde{x}(t)=A(t) e^{-i \theta(t)}
$$

where $\tilde{x}(t)$ is the Hilbert transform of $x(t)$. The amplitude function $A(t)$ and phase angle function $\theta(t)$ can be expressed as

$$
\begin{aligned}
& A(t)=A_{0} e^{-\xi \omega_{0} t} \\
& \theta(t)=\omega_{d} t+\phi_{0} .
\end{aligned}
$$

By introducing logarithm and differential operator, (12) yields

$$
\begin{aligned}
\ln A(t) & =\ln A_{0}-\xi \omega_{0} t \\
\omega(t) & =\frac{d \theta(t)}{d t}=\omega_{d} .
\end{aligned}
$$

Equation (13) shows that the damping frequency is calculated from the slope of phase angle function. The slope of amplitude function $\xi \omega_{0}$ can also be obtained. Thus, the frequency $\omega_{0}$ and damping ratio $\xi$ are calculated from $\omega_{d}=$ $\omega_{0} \sqrt{1-\xi^{2}}$.

3.2. Stochastic Subspace Identification. SSI is a robust time domain modal identification approach. Many successful applications based on SSI have been reported. The stochastic state space model is described as

$$
\begin{gathered}
x_{k+1}=A x_{k}+w_{k} \\
y_{k}=C x_{k}+v_{k},
\end{gathered}
$$

where $x_{k}$ is the state vector; $y_{k}$ is the output vector; $w_{k}$ and $v_{k}$ denote the modelling inaccuracies and measuring errors, respectively; $A$ is the state matrix and $C$ output matrix. The main objective of SSI is to identify $A$ and $C$ from outputonly response. The modal parameters can be obtained by applying eigenvalue decomposition to $A$. Based on the state space model, there are mainly two implementations of SSI algorithm: the first one directly starts from the sampled vibration data, namely, data driven SSI (SSI-data) and the other one from the covariance of the vibration data which is called covariance driven SSI (SSI-cov). Both SSI-data and SSI-cov reveal similar results with satisfying accuracy, while SSI-cov provides a better way to estimate the variance of the identified modal parameters.

The idea of combining EMD and SSI for modal identification was first proposed by Peeters and De Roeck [21]. Since the IMFs are single component signals, the stabilization diagram obtained from EMD-SSI method only contains one stable pole, which will greatly reduce the human interaction work during postprocessing. However, the EMD-SSI method depends largely on band-pass filter, which is introduced to deal with the mode mixing problem of EMD. This means one needs a priori knowledge (frequency components) of the vibration response before modal analysis. With the improved EMD, the problem of mode mixing can be solved without the so-called a priori knowledge of the signals. This paper will use SSI-cov algorithm together with IMFs extracted by bandwidth restricted EMD to identify modal parameters.

\section{Application: Output-Only Modal Analysis of a Railway Bridge}

To illustrate the effectiveness of the proposed method, output-only modal analysis of a railway bridge is performed in this section. The modal identification results from PeakPicking and SSI-cov are also listed for comparison. Before modal analysis, some basic information about the railway bridge and the ambient vibration test are introduced.

4.1. Bridge Description. Songtoujiang bridge (Figure 6) is a four span prestressed concrete bridge located at the mountain area in Jiangxi province, China. It is a continuous box girder bridge with a span arrangement of $(60+2 \times 100+60) \mathrm{m}$. One 

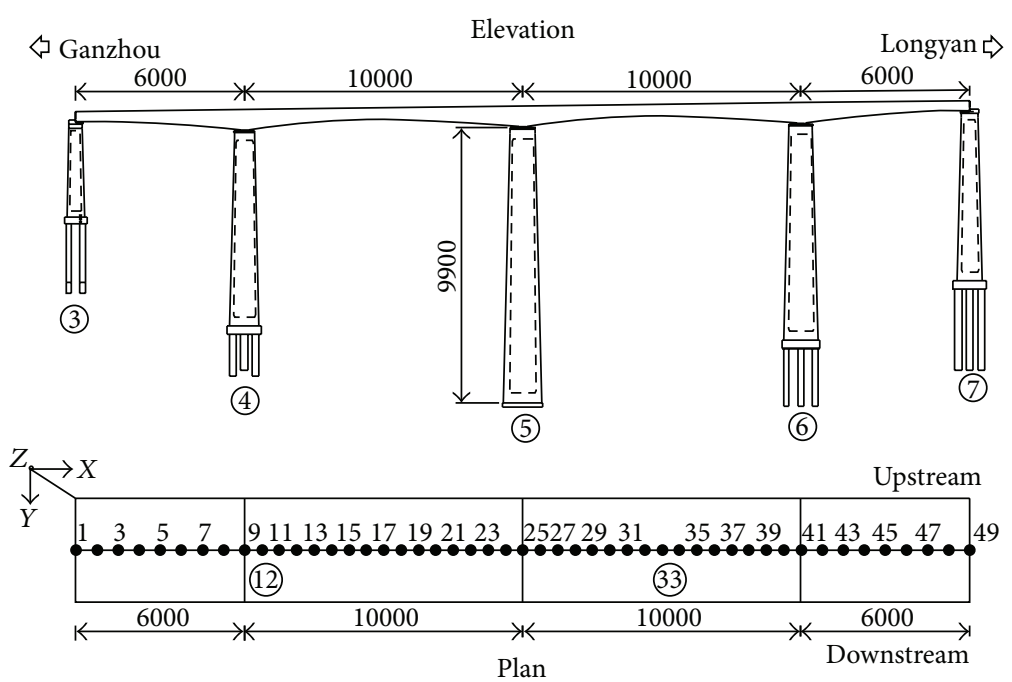

FIGURE 6: Elevation, plan, and layout of measuring points in ambient vibration test. The circles indicate reference nodes (dimensions are in centimeter).
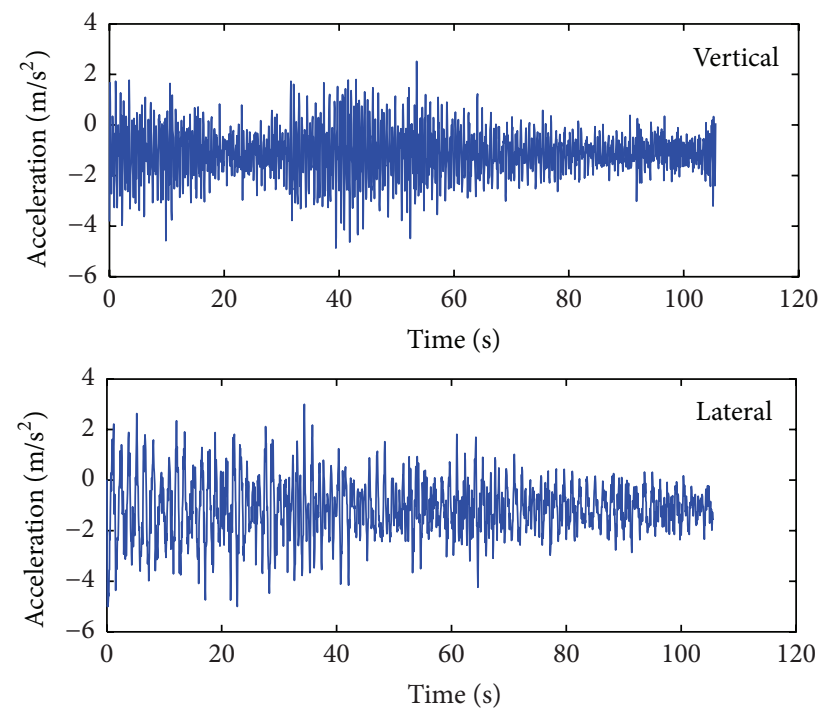

FIGURE 7: Vertical and lateral acceleration responses recorded at measuring point 5 .

of its piers is as high as $99 \mathrm{~m}$. The width of the bridge deck is $12.5 \mathrm{~m}$, on which located two opposite parallel railway lines.

4.2. Ambient Vibration Test. In order to know its dynamic properties and also gather useful information for future establishment of long-term SHM system, an ambient vibration test campaign of Songtoujiang bridge was performed. The test campaign was based on a low-cost data acquisition system with 6 channels. In total, 3 accelerometers were used for a multi-setups test with 2 reference nodes and 47 roving measuring points. As shown in Figure 6, all measuring points were placed at the center of the bridge deck, and the number in circles shows the location of reference sensors.
In each setup, vertical and lateral accelerations of bridge deck at two reference sensors and one roving sensor were recorded. The sampling frequency is $20 \mathrm{~Hz}$ and the sampling time is around 5 minutes for every setup. Figure 7 shows typical measured acceleration time histories at test point 5 . Before output-only modal analysis was performed, the test signals were first preprocessed with (1) DC components of the test signal were removed. (2) Low-pass filter was applied to test signals in order to remove high frequency noises.

4.3. Output-Only Modal Analysis. In this section, the outputonly modal analysis of Songtoujiang bridge is performed. In total, three methods are used for comparison: Peak-Picking, SSI-cov, and the improved EMD based modal identification method.

Peak-Picking is a frequency domain method aimed at determining the Frequency Response Function (FRF) of a structure. The peaks in the FRF figure represent the eigenfrequencies and the damping ratios are estimated by half-power spectrum bandwidth method. FRF is estimated by the input and output measurements of the structure. In the outputonly case, the FRF cannot be determined but only instead by the Fourier spectrum of the output response. Figure 8 shows the vertical and lateral spectrum of acceleration responses recorded at measuring point 5 .

Figure 9 plots the stabilization diagram of setup 5 obtained from SSI-cov algorithm. There are many stable poles in the figure. One need to decide which stable poles represent system modes, which are spurious modes. This kind of human interaction could be very time-consuming; especially there are 47 stabilization diagrams in total. Besides some stable poles are quite close, it is hard to choose system modes. For example, there are two stable poles near $1.5 \mathrm{~Hz}$; both of them could be system poles.

For the improved EMD method, first the acceleration responses should be decomposed into IMFs. Take the lateral acceleration response at measuring point 5 as an example. 


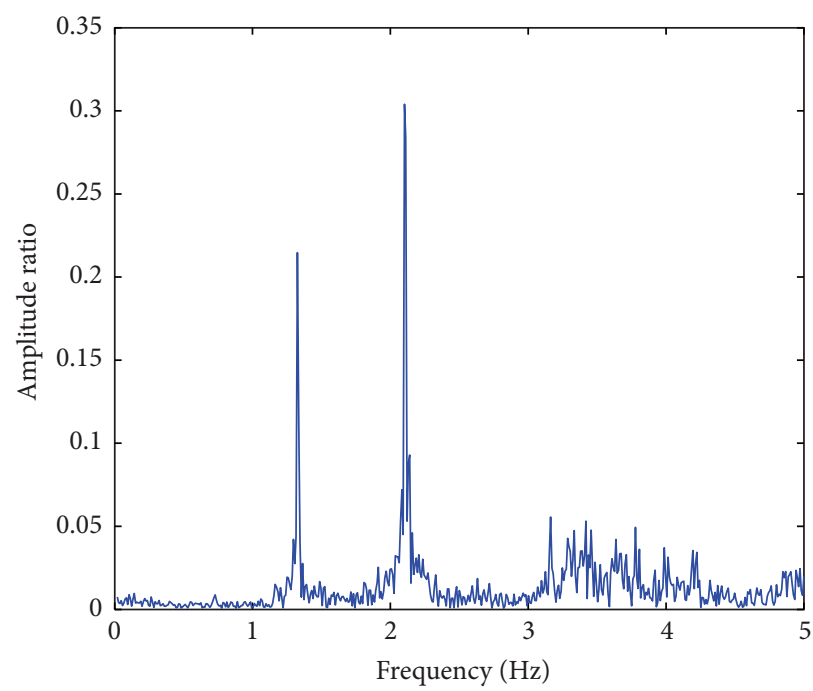

(a)

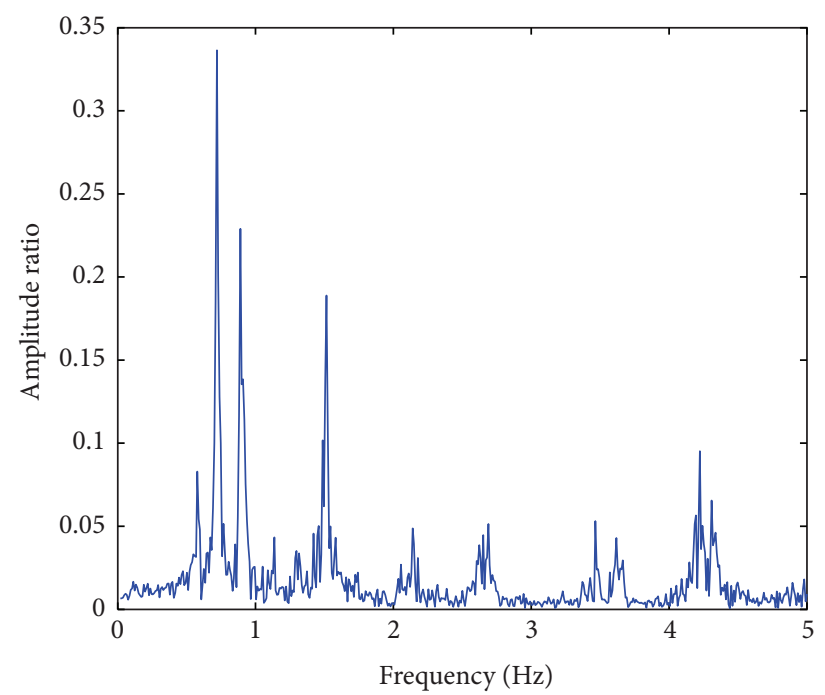

(b)

FIgURE 8: The Fourier spectrum of the accelerations shown in Figure 4. (a) Vertical spectrum; (b) lateral spectrum.

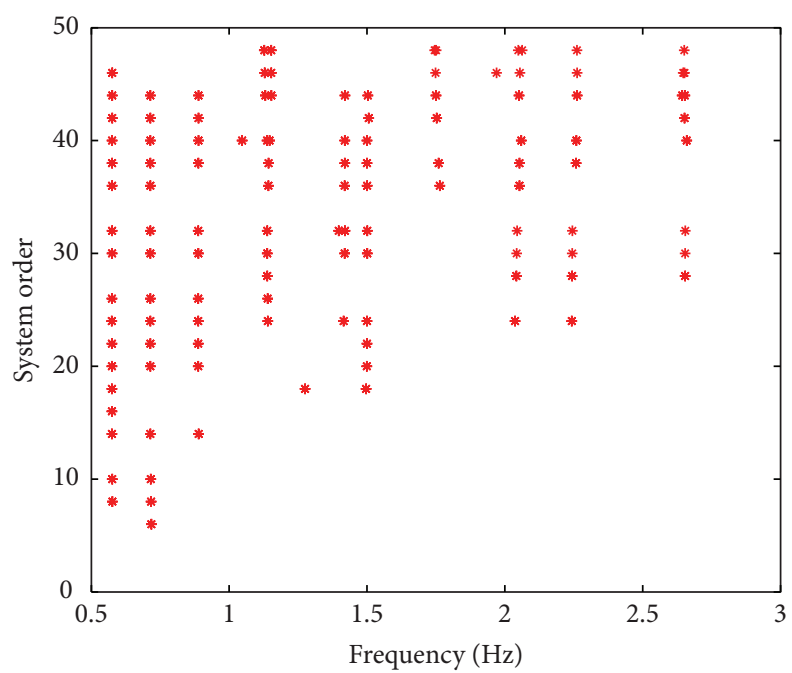

Figure 9: The stabilization diagram obtained from conventional SSI-cov. The stars denote the stable points with following stable criterion: $d f_{i} \leq 1 \%, d \xi_{i} \leq 5 \%$, and $d \mathrm{MAC}_{i} \leq 1 \%$. MAC is the Modal Assurance Criterion value of two adjacent mode shapes.

Figure 10 shows the first six IMFs of the lateral acceleration response. It is shown that the frequencies of the IMFs are decreasing. After the IMFs were extracted, the modal parameter can be identified by either RDT or SSI-cov.

Figure 11 shows the free decay response extracted from IMF by RDT. The corresponding amplitude function and phase angle function are shown in Figure 12. Through least square fitting, the slopes of the amplitude function and phase angle function were calculated. The slope of amplitude function $-\xi \omega_{0}=-9.473 \mathrm{rad} / \mathrm{s}$. The slope of phase angle function $\omega_{d}=\omega_{0} \sqrt{1-\xi^{2}}=0.102$. Solving the two equations simultaneously yields the frequency $\omega_{0}=1.509 \mathrm{~Hz}$ and the damping ratio $\xi=0.068$. This mode is the fourth lateral bending mode. Following the same rules, the frequencies and damping ratios of the left modes can be obtained.

Assemble the IMFs contain the same frequency component from different setups and through SSI-cov algorithm, the stabilization diagrams in Figure 13 were obtained. In these stabilization diagrams, there is only one stable pole. The spurious modes are separated into some discrete stable points. The system modes can be easily determined. The Fourier spectrum of each IMF is also shown in the stabilization diagram which further illustrated that IMF obtained from the improved EMD is single frequency component signal.

4.4. Discussion. Table 1 shows the modal parameters of Songtoujiang Bridge identified from different approaches. The experimental results from PP, SSI-cov, and standard EMD are listed for comparison. The analytical results from a preliminary Finite Element Model (FEM) are also listed for comparison. In total, four lateral bending modes and two vertical bending modes can be extracted from operational vibration measurements. Because of the relatively low sampling frequency and short sampling time of the low-cost data acquisition system, there might exist some inaccuracies in the higher modes. From Table 1, it is shown that the experimental results from different methods show good agreement, while the analytical results show some deviations especially in 2nd and 3rd lateral bending mode. This means that the preliminary FE model need to be updated. A higher accuracy of frequencies is found than the damping ratios. Due to mode mixing, the third lateral bending mode cannot be extracted from standard EMD. With the improved EMD method, the natural frequencies and damping ratios can be extracted with satisfactory accuracy.

The main contribution of the proposed improved EMD based modal identification technique is that it provide more reasonable theory explanations to deal with nonstationary 

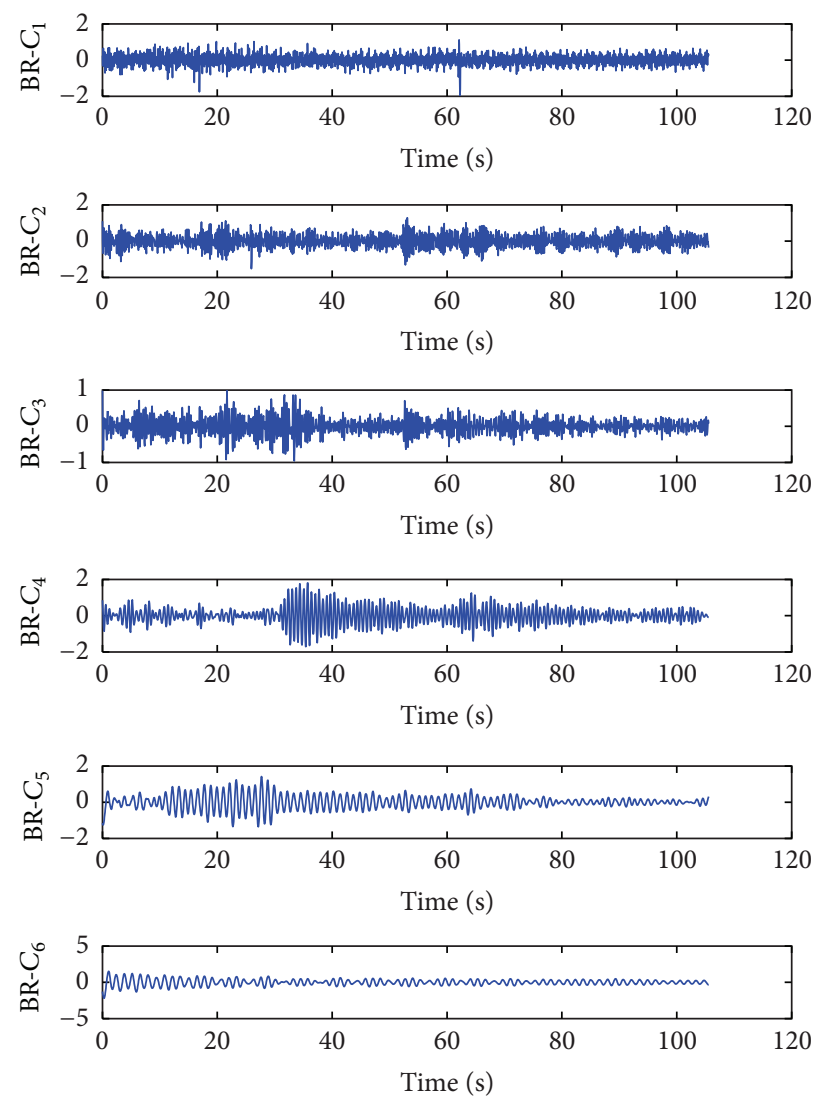

FIGURE 10: The first six IMFs obtained by applying bandwidth restricted EMD to the lateral acceleration at measuring point 5.

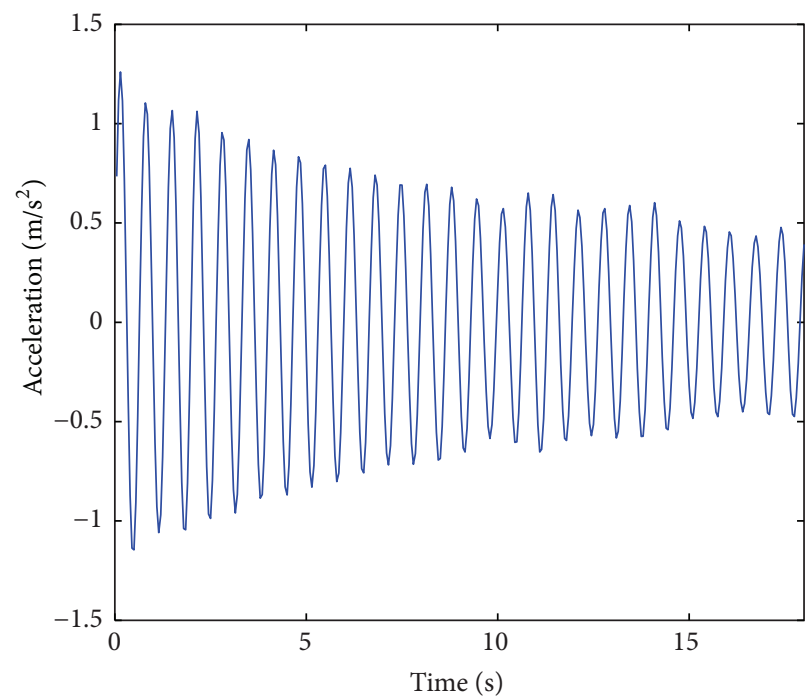

Figure 11: The free decay response extracted from IMF (BR- $C_{4}$ in Figure 10) by random decrement technique.

excitations (ambient loads) and measurements (dynamic responses) without assuming them to be stationary processes. Although some subspace algorithms were reported to have constant estimates of eigenstructures even under nonstationary excitations $[30,31]$, the theory derivation of subspace algorithms is based on stationary inputs assumption. However, the EMD is proved to be very suitable for nonstationary signals, even nonlinear signals. Another contribution of the proposed method is that band-pass filter, which is often employed to deal with closely spaced modes, is no longer 


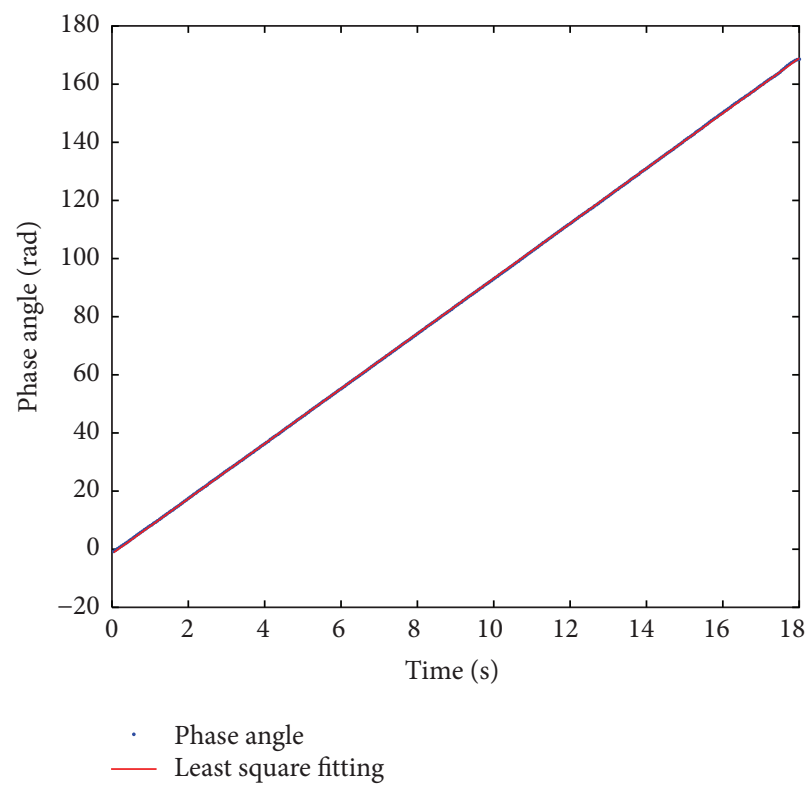

(a)

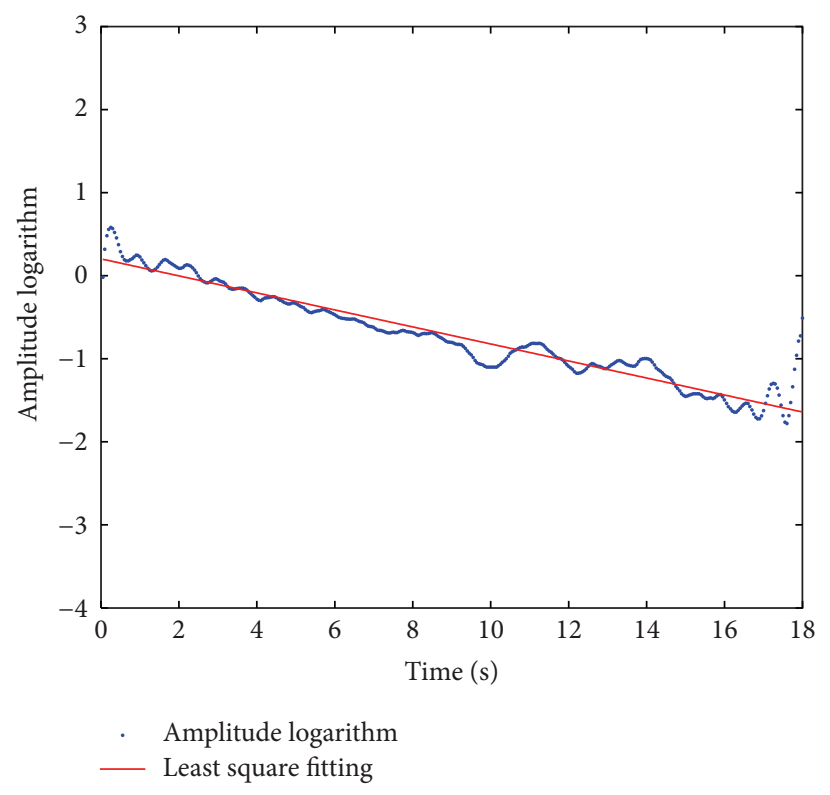

(b)

FIgURE 12: Phase angle and amplitude function of the free decay response in Figure 6. (a) Phase angle and its least square fitting. (b) The amplitude logarithm and its least square fitting.

TABLE 1: The comparison of the modal parameters obtained from different algorithms.

\begin{tabular}{|c|c|c|c|c|c|c|c|c|c|c|}
\hline \multirow{3}{*}{ Mode } & \multirow{3}{*}{$\begin{array}{l}\text { fem } \\
f_{f}(\mathrm{~Hz})\end{array}$} & \multirow{3}{*}{$\begin{array}{l}\mathrm{PP} \\
f_{\mathrm{PP}}(\mathrm{Hz})\end{array}$} & \multirow{2}{*}{\multicolumn{2}{|c|}{ SSI-cov }} & \multirow{2}{*}{\multicolumn{2}{|c|}{ Std. EMD-RDT }} & \multicolumn{4}{|c|}{ Improved EMD } \\
\hline & & & & & & & \multicolumn{2}{|c|}{ with RDT } & \multicolumn{2}{|c|}{ with SSI-cov } \\
\hline & & & $f_{\mathrm{SSI}}(\mathrm{Hz})$ & $\xi_{\text {SSI }}(\%)$ & $f_{e}(\mathrm{~Hz})$ & $\xi_{e}(\%)$ & $f_{e r}(\mathrm{~Hz})$ & $\xi_{\text {er }}(\%)$ & $f_{e s}(\mathrm{~Hz})$ & $\xi_{e s}(\%)$ \\
\hline $1 s t \leftrightarrow$ & 0.662 & 0.58 & 0.583 & 2.74 & 0.530 & 7.41 & 0.530 & 3.12 & 0.583 & 2.28 \\
\hline $2 \mathrm{nd} \leftrightarrow$ & 0.789 & 0.71 & 0.721 & 3.63 & 0.714 & 7.23 & 0.714 & 2.85 & 0.716 & 3.52 \\
\hline $3 \mathrm{rd} \leftrightarrow$ & 1.137 & 0.89 & 0.914 & 3.91 & $\mathrm{n} / \mathrm{a}$ & $\mathrm{n} / \mathrm{a}$ & 0.916 & 1.96 & 0.915 & 4.33 \\
\hline 1st $\uparrow$ & 1.333 & 1.32 & 1.302 & 1.54 & 1.319 & 1.28 & 1.331 & 1.68 & 1.325 & 2.48 \\
\hline 4 th $\leftrightarrow$ & 1.570 & 1.50 & $1.481 / 1.508$ & $1.65 / 1.26$ & 1.505 & 2.84 & 1.509 & 1.37 & 1.512 & 1.03 \\
\hline 2nd $\uparrow$ & 1.975 & 2.09 & 1.996 & 4.82 & 2.097 & 0.83 & 1.99 & 4.23 & 1.983 & 3.68 \\
\hline
\end{tabular}

Note: $\leftrightarrow$ denotes lateral bending mode; $\uparrow$ denotes vertical bending mode; $n / a:$ not a number.

needed with bandwidth restricted EMD. The application example demonstrates that quite accurate modal parameters can be obtained by the proposed method, meaning that the improved EMD based modal identification technique can serve as an alternative to existing output-only modal analysis methods.

\section{Conclusions}

Starting from the nonstationary vibration measurements and closely spaced mode of bridge structures, this paper has developed a bandwidth restricted EMD for output-only modal analysis. Specifically, the mode mixing problem of standard EMD when dealing with closely spaced modes has been effectively restrained by the improved EMD. Through numerical simulation and application in a railway bridge, the following conclusions have been made:
(1) The bandwidth restricted EMD have restrained the problem of mode mixing, making sure that each IMF obtained from EMD contains only one frequency component. It is based on the binary filter property of EMD and can be easily implemented, without introducing too much computational complexities. The numerical simulation have illustrated the effectiveness of the improved EMD.

(2) The modal parameters obtained through improved EMD algorithm show good agreement with those extracted from PP and SSI-cov. Compared with conventional EMD based RDT or SSI-cov modal identification algorithms, the improved EMD based method does not rely on band-pass filter when dealing with closely spaced modes, meaning that the a priori knowledge (frequency components) of nonstationary output measurements is not needed. 


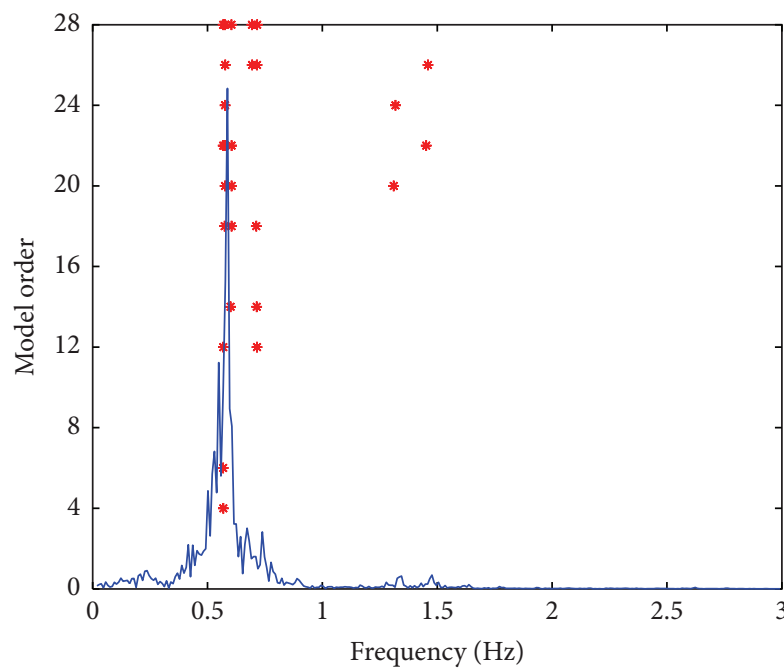

(a)

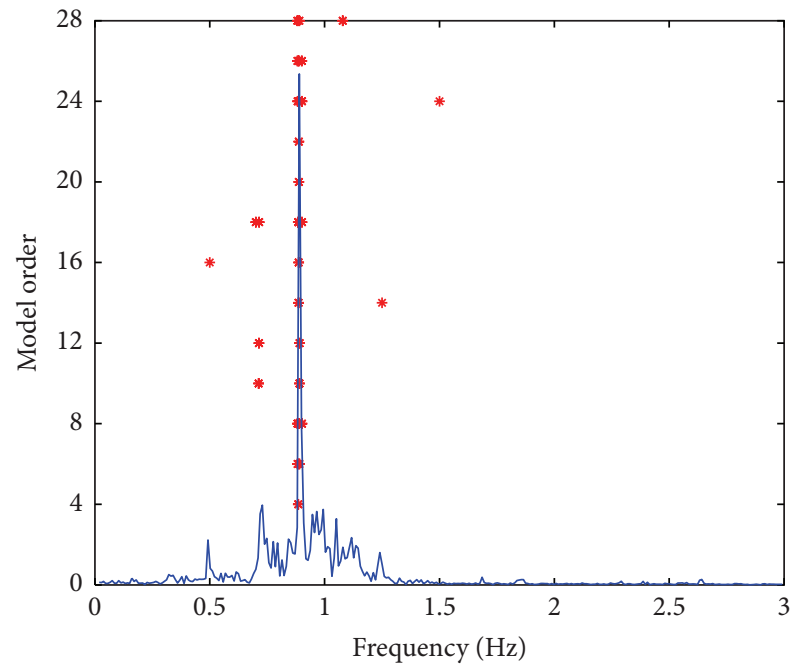

(c)

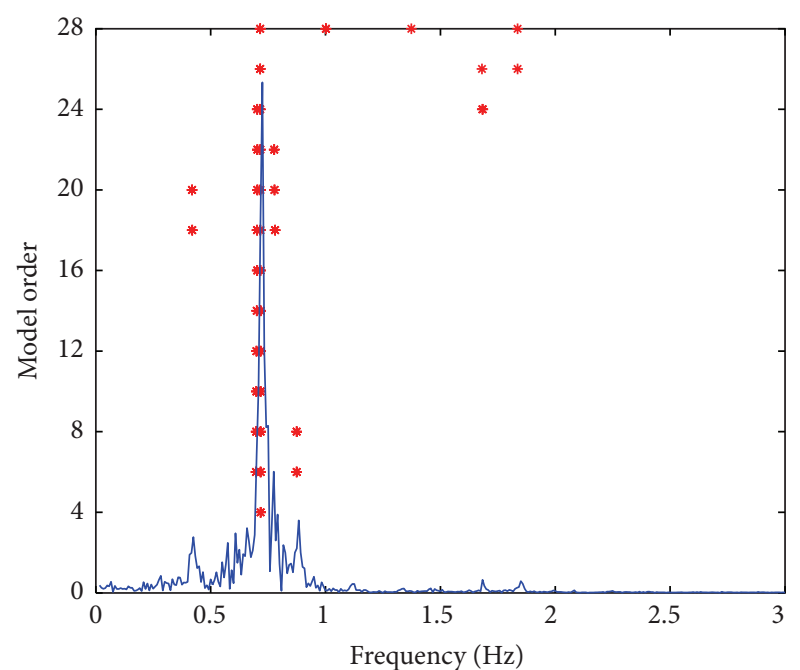

(b)

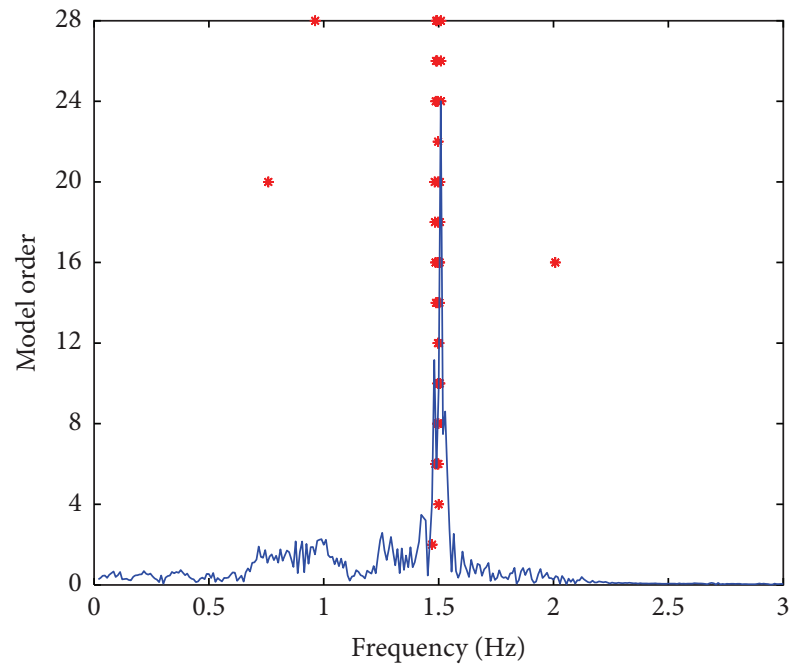

(d)

FIGURE 13: The stabilization diagrams obtained from bandwidth restricted EMD based SSI-cov algorithm. (a) Mode 1: $f=0.583 \mathrm{~Hz}$; (b) mode 2: $f=0.716 \mathrm{~Hz}$; (c) mode 3: $f=0.915 \mathrm{~Hz}$; (d) mode 5: $f=1.512 \mathrm{~Hz}$. The stars denote the stable points with following stable criterion: $d f_{i} \leq 1 \%, d \xi_{i} \leq 5 \%$, and $d \mathrm{MAC}_{i} \leq 1 \%$. The fourier spectrums are scaled to sever as comparison.

\section{Conflict of Interests}

The authors declare that there is no conflict of interests regarding the publication of this paper.

\section{Acknowledgments}

Financial support from National Natural Science Foundation of China (Grant no. 51108382), Natural Science Foundation of Hubei Province (Grant no. 2015CFB393), and the Fundamental Research Foundation of Central Universities (Grant no. 2014-IV-047) is greatly acknowledged.

\section{References}

[1] K. H. Hsieh, M. W. Halling, and P. J. Barr, "Overview of vibrational structural health monitoring with representative case studies," Journal of Bridge Engineering, vol. 11, no. 6, pp. 707-715, 2006.

[2] J. M. Ko and Y. Q. Ni, “Technology developments in structural health monitoring of large-scale bridges," Engineering Structures, vol. 27, no. 12, pp. 1715-1725, 2005.

[3] B. Peeters and G. De Roeck, "One-year monitoring of the Z24bridge: environmental effects versus damage events," Earthquake Engineering \& Structural Dynamics, vol. 30, no. 2, pp. 149171, 2001. 
[4] S. Soyoz and M. Q. Feng, "Long-term monitoring and identification of bridge structural parameters," Computer-Aided Civil and Infrastructure Engineering, vol. 24, no. 2, pp. 82-92, 2009.

[5] F. Magalhães, Á. Cunha, and E. Caetano, "Dynamic monitoring of a long span arch bridge," Engineering Structures, vol. 30, no. 11, pp. 3034-3044, 2008.

[6] Z. H. Zong, T. L. Wang, and T. Z. Huang, "State-of-the-art of bridge health monitoring," Journal of Fuzhou University (Natural Science Edition), vol. 30, pp. 127-152, 2002.

[7] M. M. Abdel Wahab and G. De Roeck, "Damage detection in bridges using modal curvatures: application to a real damage scenario," Journal of Sound and Vibration, vol. 226, no. 2, pp. 217-235, 1999.

[8] Z. F. Zhang, K. Shankar, T. Ray, E. V. Morozov, and M. Tahtali, "Vibration-based inverse algorithms for detection of delamination in composites," Composite Structures, vol. 102, pp. 226-236, 2013.

[9] B. Jaishi, H.-J. Kim, M. K. Kim, W.-X. Ren, and S.-H. Lee, "Finite element model updating of concrete-filled steel tubular arch bridge under operational condition using modal flexibility," Mechanical Systems and Signal Processing, vol. 21, no. 6, pp. 2406-2426, 2007.

[10] E. Reynders, A. Teughels, and G. De Roeck, "Finite element model updating and structural damage identification using OMAX data," Mechanical Systems and Signal Processing, vol. 24, no. 5, pp. 1306-1323, 2010.

[11] M. I. Friswell and J. E. Mottershead, Finite Element Model Updating in Structural Dynamics, Kluwer Academic, Dordrecht, The Netherlands, 1995.

[12] A. Teughels and G. De Roeck, "Structural damage identification of the highway bridge Z24 by FE model updating," Journal of Sound and Vibration, vol. 278, no. 3, pp. 589-610, 2004.

[13] C. Gentile and N. Gallino, "Condition assessment and dynamic system identification of a historic suspension footbridge," Structural Control and Health Monitoring, vol. 15, no. 3, pp. 369-388, 2008.

[14] J. S. Bendat and A. G. Piersol, Engineering Applications of Correlation and Spectral Analysis, John Wiley \& Sons, New York, NY, USA, 2nd edition, 1993.

[15] R. Brincker, L. Zhang, and P. Andersen, "Modal identification of output-only systems using frequency domain decomposition," Smart Materials and Structures, vol. 10, no. 3, pp. 441-445, 2001.

[16] B. Peeters, H. Van der Auweraer, P. Guillaume, and J. Leuridan, "The PolyMAX frequency-domain method: a new standard for modal parameter estimation?" Shock and Vibration, vol. 11, no. 3-4, pp. 395-409, 2004.

[17] X. He and G. De Roeck, "System identification of mechanical structures by a high-order multivariate autoregressive model," Computers \& Structures, vol. 64, no. 1-4, pp. 341-351, 1997.

[18] J. M. Caicedo, "Practical guidelines for the natural excitation technique (NExT) and the eigensystem realization algorithm (ERA) for modal identification using ambient vibration," Experimental Techniques, vol. 35, no. 4, pp. 52-58, 2011.

[19] Á. Cunha and E. Caetano, "Experimental modal analysis of civil engineering structures," Sound and Vibration, vol. 40, no. 6, pp. 12-20, 2006.

[20] P. Mohanty and D. J. Rixen, "A modified Ibrahim time domain algorithm for operational modal analysis including harmonic excitation," Journal of Sound and Vibration, vol. 275, no. 1-2, pp. 375-390, 2004.
[21] B. Peeters and G. De Roeck, "Reference-based stochastic subspace identification for output-only modal analysis," Mechanical Systems and Signal Processing, vol. 13, no. 6, pp. 855-878, 1999.

[22] J. N. Yang, Y. Lei, S. W. Pan, and N. Huang, "System identification of linear structures based on Hilbert-Huang spectral analysis. Part 1: normal modes," Earthquake Engineering \& Structural Dynamics, vol. 32, no. 9, pp. 1443-1467, 2003.

[23] J. N. Yang, Y. Lei, S. W. Pan, and N. Huang, "System identification of linear structures based on Hilbert-Huang spectral analysis. Part 2: complex modes," Earthquake Engineering \& Structural Dynamics, vol. 32, no. 10, pp. 1533-1554, 2003.

[24] X. H. He, X. G. Hua, Z. Q. Chen, and F. L. Huang, "EMD-based random decrement technique for modal parameter identification of an existing railway bridge," Engineering Structures, vol. 33, no. 4, pp. 1348-1356, 2011.

[25] D.-J. Yu and W.-X. Ren, "EMD-based stochastic subspace identification of structures from operational vibration measurements," Engineering Structures, vol. 27, no. 12, pp. 1741-1751, 2005.

[26] B. Yan and A. Miyamoto, "A comparative study of modal parameter identification based on wavelet and Hilbert-Huang transforms," Computer-Aided Civil and Infrastructure Engineering, vol. 21, no. 1, pp. 9-23, 2006.

[27] Z. H. Wu and N. E. Huang, "Ensemble empirical mode decomposition: a noise-assisted data analysis method," Advances in Adaptive Data Analysis, vol. 1, no. 1, pp. 1-41, 2009.

[28] R. Deering and J. F. Kaiser, "The use of a masking signal to improve empirical mode decomposition," in Proceedings of the IEEE International Conference on Acoustics, Speech, and Signal Processing (ICASSP '05), vol. 4, pp. iv/485-iv/488, IEEE, Orlando, Fla, USA, March 2005.

[29] N. E. Huang, Z. Shen, S. R. Long et al., "The empirical mode decomposition and the Hilbert spectrum for nonlinear and non-stationary time series analysis," The Royal Society of London. Proceedings A. Mathematical, Physical and Engineering Sciences, vol. 454, no. 1971, pp. 903-995, 1998.

[30] L. Mevel, M. Basseville, A. Benveniste, and M. Goursat, "Merging sensor data from multiple measurement set-ups for nonstationary subspace-based modal analysis," Journal of Sound and Vibration, vol. 249, no. 4, pp. 719-741, 2002.

[31] M. Basseville, L. Mevel, and M. Goursat, "Statistical modelbased damage detection and localization: subspace-based residuals and damage-to-noise sensitivity ratios," Journal of Sound and Vibration, vol. 275, no. 3-5, pp. 769-794, 2004. 

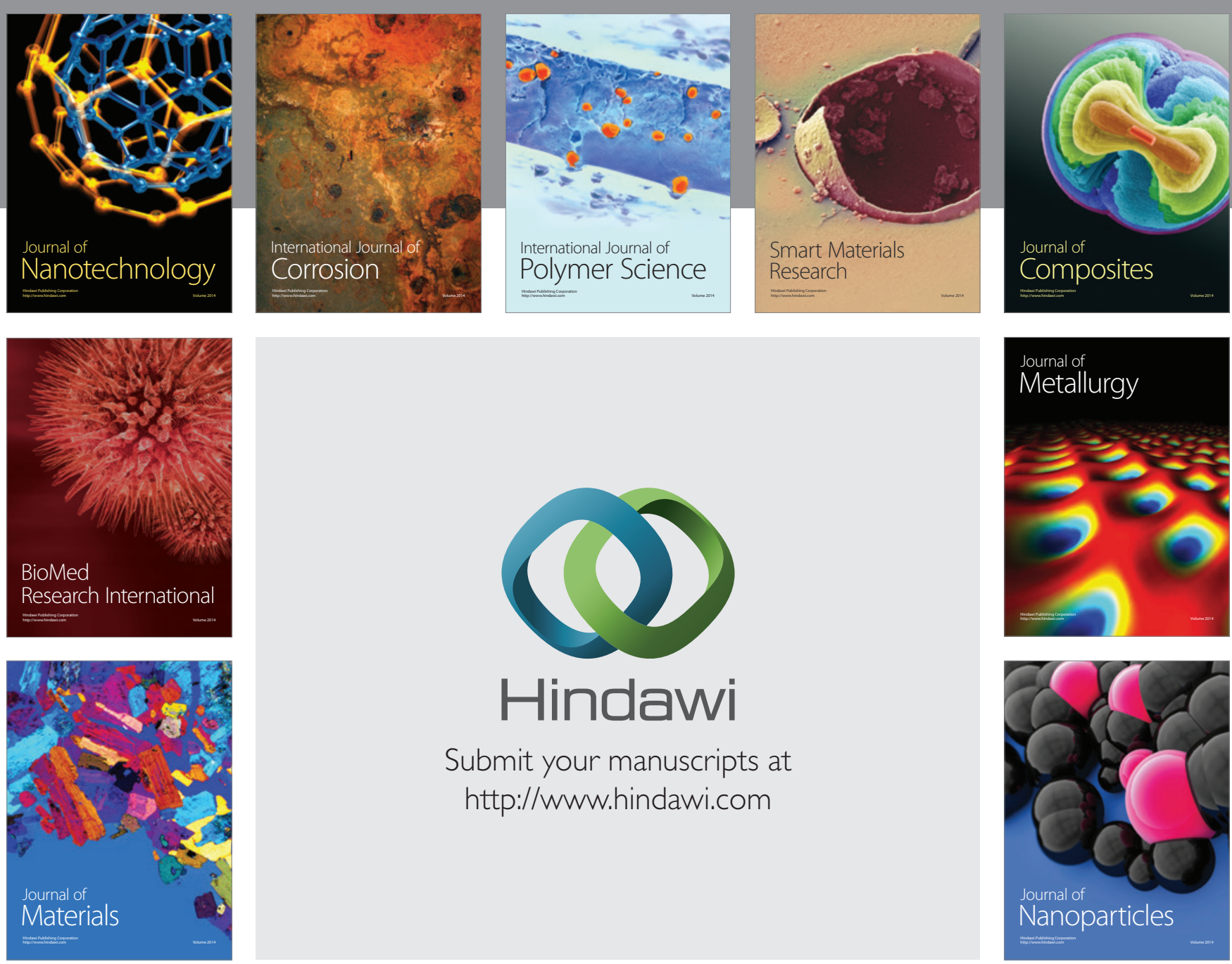

Submit your manuscripts at http://www.hindawi.com
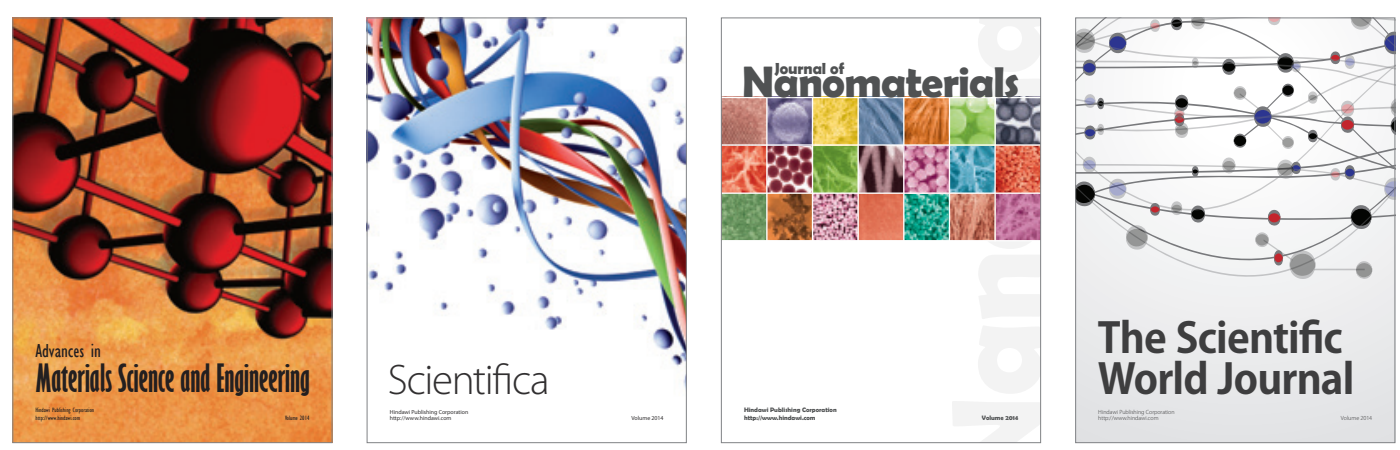

\section{The Scientific World Journal}
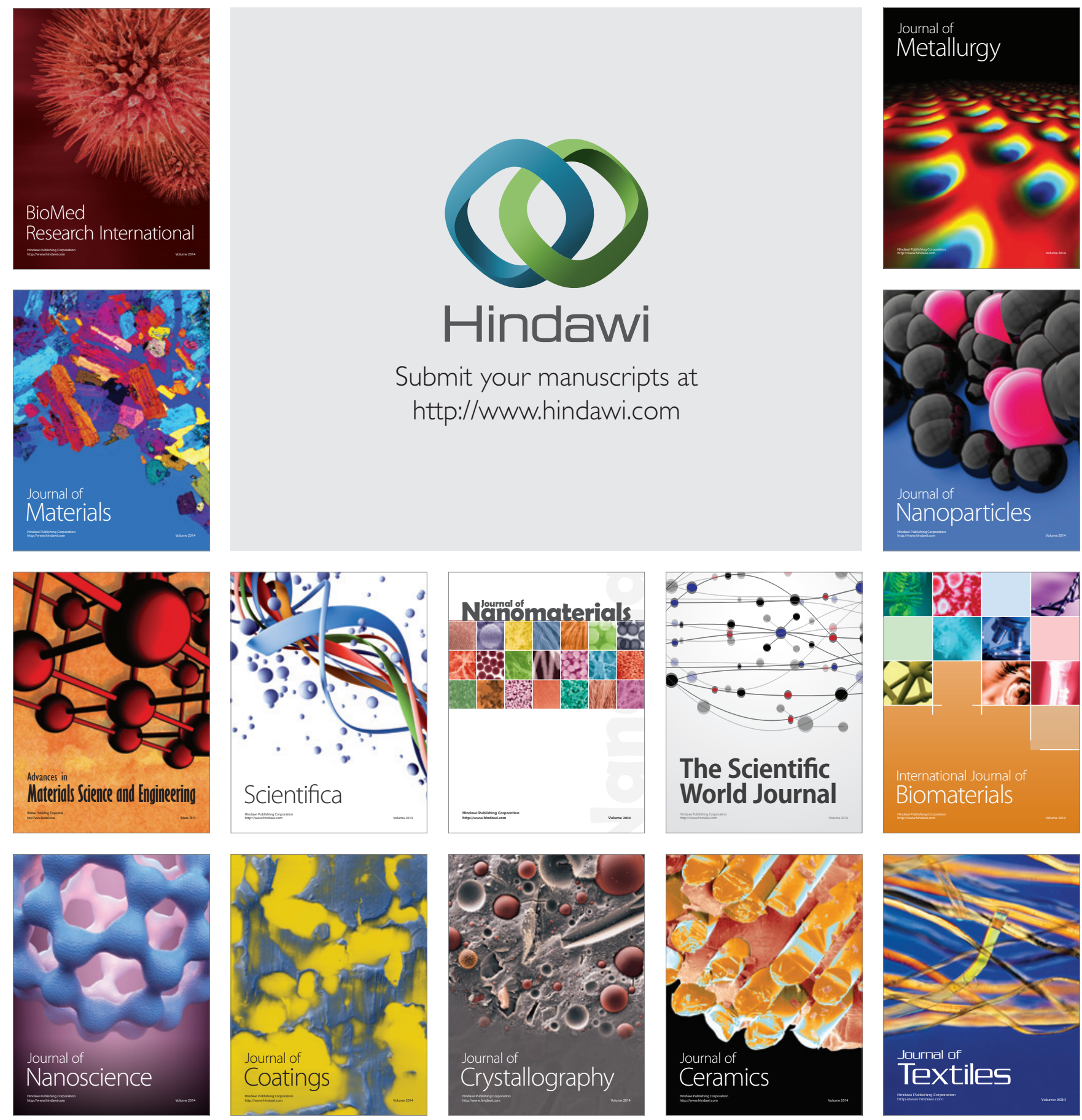